\title{
Proline-Rich Protein 11 Regulates Self- Renewal and Tumorigenicity of Gastric Cancer Stem Cells
}

\author{
Hongtao $\mathrm{Hu}^{\mathrm{a}}$ Zongchang Song ${ }^{\mathrm{b}} \quad$ Quanjun Yao $^{\mathrm{a}} \quad \mathrm{Xiang}_{\mathrm{Geng}}{ }^{\mathrm{a}} \quad \mathrm{Li}_{\mathrm{J}} \mathrm{Jiang}^{\mathrm{a}}$ \\ Chenyang Guo Hailiang Lia \\ aDepartment of Interventional Radiology, The Affiliated Cancer Hospital of Zhengzhou University/ \\ Henan Cancer Hospital. Zhengzhou, 'Department of Radiotherapy, Zhebei Mingzhou Hospital, Huzhou, \\ China
}

\section{Key Words}

Cancer stem cells • Gastric cancer $\bullet$ Proline-rich protein 11

\begin{abstract}
Background/Aims: Gastric cancer is a highly aggressive tumor containing cancer stem cells (CSCs), which participate in tumor initiation, therapeutic resistance, and tumor relapse. Prolinerich protein 11 (PRR11) has been shown to be up-regulated in human cancers; however, its role in gastric CSCs is unknown. We hypothesize that PRR11 may affect tumorigenicity of gastric CSCs. In this study, we explored the biological function and regulation of PRR11 in gastric CSCs. Methods: Expression of PRR11 was evaluated in gastric CSC cell line by real-time quantitative PCR and western blot. The effect of PRR11 on tumorigenicity was examined by interference with gene expression using lentiviral vector-loaded shRNA. A xenograft tumor model using NOD/SCID mice was established to examine the role of PRR11 in tumor development. Results: Data showed that PRR11 was highly expressed in gastric CSCs. PRR11 was responsible for the maintenance of self-renewal and tumorigenicity of gastric CSCS, and overexpression of exogenous PRR11 could restore the self-renewal of gastric non-CSCs. Furthermore, interference with PRR11 altered the expression of stemness transcription factors. Interestingly, MAPK signaling controlled PRR11 expression by increasing PRR11 protein stability, and maintained gastric CSCs self-renewal in a PRR11 dependent manner. Conclusions: PRR11 regulated selfrenewal and tumorigenicity of gastric CSCs through MAPK signaling, and could be used as a therapeutic target for gastric cancer.




\section{Cellular Physiology Cell Physiol Biochem 2018;47:1721-1728 and Biochemistry Published online: June 28, $2018 \quad \begin{aligned} & \text { DO } 2018 \text { The Author(s). Published by S. Karger AG, Basel } \\ & \text { www.karger.com/cpb }\end{aligned}$ \\ Hu et al.: PRR11 and Gastric Cancer Stem Cells}

\section{Introduction}

Gastric carcinoma (GC) is the fourth most common malignancy worldwide [1]. Although there have been important medical advances in the diagnosis and treatment of GC over the past few decades, the survival rate remains low [1-4]. Novel treatment strategies are urgently needed.

Recently studies have demonstrated that a subpopulation of cancer cells, often referred to as cancer stem cells (CSCs) or tumor initiating cells (TICs) [5], is capable of extensive proliferation, self-renewal, and increased frequency of tumor initiation [6]. Gastric CSCs can be enriched with several defined markers, including CD44, aldehyde dehydrogenase (ALDH), and Lgr $5[7,8]$. Stemness markers such as SOX 2 and Oct-3/4 have also been accepted for the identification of gastric CSCs [9]. However, the key components and molecular mechanisms contributing to self-renewal and tumorigenicity of gastric CSCs are largely unknown.

The proline-rich protein 11 (PRR11) was identified as a novel gene and has an important role in both cell cycle progression and tumorigenesis [10]. PRR11 has been indicated as a potential novel target in the diagnosis and treatment of human lung cancer [11]. Through regulating important genes involved in cell cycles and tumorigenesis, PRR11 participates in the initiation and progression of lung cancer and epithelial-to-mesenchymal transition in breast cancer [11]. Our previous research showed that PRR11 widely activated in human gastric cancer, and was an independent prognostic factor for gastric cancer patients, which are consistent with the hypothesis that PRR11 functions as an oncogene in the development and progression of gastric cancer [12]. However, whether an increase in PRR11 expression is responsible for the expansion of gastric CSC populations is not known.

The objective of the study was to investigate the effect of PRR11 on gastric CSCs. We found that PRR11 participated in the self-renewal and tumorigenicity of gastric CSCs. We also found that MAPK signaling activity played an important role in maintaining PRR11 expression through increased PRR11 protein stability to maintain gastric CSCs self-renewal.

\section{Materials and Methods}

\section{Cell Culture}

Human gastric cancer cell lines (SGC-7901 and HGC-27) were purchased from Cell Bank, Chinese Academy of Sciences. All cells were grown in Dulbecco's modified Eagle's medium (Invitrogen) supplemented with $10 \%$ fetal bovine serum (Gibco) at $37^{\circ} \mathrm{C}$ in a humidified atmosphere containing $5 \% \mathrm{CO}_{2}$.

\section{Colony Formation}

For analysis of colony formation, SGC-7901 and HGC-27 cells were thoroughly dissociated with $0.25 \%$ trypsin in $0.02 \%$ EDTA (Sigma, USA) to prepare single cell suspension. The cells were then plated in 6-well plates at a density of 200 cells/well in DMEM containing $10 \%$ FBS. After incubation for 2 weeks at $37^{\circ} \mathrm{C}$, colonies containing more than 50 cells were counted after Giemsa staining.

\section{Sphere Formation}

For the sphere formation assay, 100 cells were seeded into Costar Ultra Low Cluster 24-well plates (Corning) and cultured in DMEM/F12 medium (Sigma) supplemented with 5ng/ml epidermal growth factor (EGF, sigma, USA), B27 supplement (1X, Invitrogen, USA), 20ng/mL basic fibroblast growth factor (Peprotech), and $10 \mathrm{ng} / \mathrm{mL}$ hepatocyte growth factor (Peprotech). Cells were incubated at $37^{\circ} \mathrm{C}$ for 2 weeks and numbers of spheres were counted.

Lentiviral vector-loaded shRNA to interfere with PRR11 expression

Lentiviral vector-loaded shRNA was used to interfere with PRR11 expression. Briefly, lentivirus expression plasmid containing small interference RNA targeting PRR11 was constructed by GENECHEM (Shanghai, China) and was used to infect the gastric CSCs in presence of $6 \mu \mathrm{g} / \mathrm{ml}$ polybrene. Cells were then selected by puromycin, and knockdown of PRR11 was confirmed by western blot. 


\section{Cellular Physiology Cell Physiol Biochem 2018;47:1721-1728

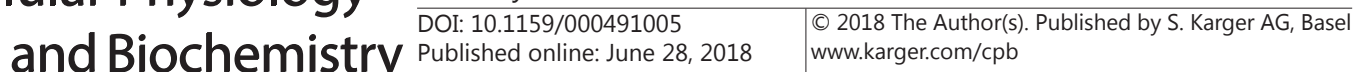

Hu et al.: PRR11 and Gastric Cancer Stem Cells

\section{Western Blot}

Total protein was prepared from lysates of SGC-7901 and HGC-27 gastric carcinoma cell lines. Western blots were performed in the standard manner using a goat polyclonal antibody against human PRR11 (dilution, 1:1000), SOX2 (dilution, 1:1000), OCT4 (dilution, 1:1000), c-Myc (dilution, 1:500), Nanog (dilution, 1:1000), GAPDH (dilution, 1:1000), and a horseradish peroxidase-conjugated anti-goat IgG antibody diluted 1:3000 as the secondary antibody. Proteins were detected by ECL detection reagent.

\section{RNA extraction and real-time PCR}

Real-time quantitative PCR reaction was performed using SYBR1 Premix Ex TaqTM kit (Takara, Kyoto, Japan) according to the manufacturer's instructions and conventional PCR assays were performed using previously described method [12]. GAPDH was used as an internal standard. The PCR cycle was performed according to previously published protocol [12]. Reactions and analysis were performed using the ABI PRISM 7300 PCR and detection system (Applied Biosystems, Carlsbad, CA). The primers used are as follow: PRR11: F: 5'- CGTATCTGCCACCGAGAACTT-3'; R: 5'-GAGATGGTCTTCAGTGCTTCCT-3'; GAPDH: F: 5'-TGACTTCAACAGCGACACCCA-3'; R: 5'-CAC CCTGTTGCTGTAGCCAAA-3'.

\section{Xenografts}

SGC-7901 cells were injected subcutaneously into 5-week-old NOD/SCID mice with implantation of $1 \times 10^{3}, 5 \times 10^{3}, 1 \times 10^{4}$, and $2 \times 10^{6}$ cells per mouse, respectively $(\mathrm{n}=5)$. At the end of 6 weeks or longer until they reached an appropriate size, mice were euthanized, and xenografts were removed and measured. Tumor volume (TV) was calculated by the formula: TV $=\mathrm{L}$ (length) $\times \mathrm{W}^{2}$ (width) $/ 2$. The usage of the mice was approved by the experimental animal ethics committee of the hospital (No. 150126).

\section{Statistical analysis}

All experiments were conducted at least three times and the results were from representative experiments. Data were expressed as the mean \pm SEM, and the statistical significance between testing and control groups was analyzed with SPSS10.0 statistical software. When two groups were compared, the unpaired Student's t test was used. $\chi 2$ test was used for analysis of in vivo xenografts. $\mathrm{P}<0.05$ was considered statistically significant.

\section{Results}

\section{PRR11 was highly expressed in gastric CSCS}

We previously reported that PRR11 was highly expressed in gastric tumor tissues, and played important roles in tumor progression [12]. However, its role in gastric CSCs is unknown. To understand the PRR11 regulated self-renewal and tumorigenicity of gastric CSCs, we firstly examined whether PRR11 expression was elevated in gastric CSCs using CD44 biomarker. Our results showed that the mRNA level $(\mathrm{P}<0.001$, Fig. $1 \mathrm{~A})$ and protein level (Fig. 1B) of PRR11 were significantly elevated in gastric CSCs compared to those in

Fig. 1. PRR11 was highly expressed in gastric CSCs (A) PRR11 was detected by quantitative real-time PCR (QRT-PCR) in gastric CSCs and non-CSCs sorted from gastric cell line SGC-7901. Results are shown as means \pm SEM of three independent experiments. $* * * \mathrm{P}<0.001$. (B) Western blotting analyzed PRR11 expression in gastric CSCs and non-CSCs sorted from gastric cell lines (SGC-7901 and HCG-27). (C) PRR11 expression was measured by QRT-PCR in sphere cells and non-sphere CSCs derived from SGC-7901. Results are shown as means \pm SEM of three independent experiments. ${ }^{* * *} \mathrm{P}<0.001$.

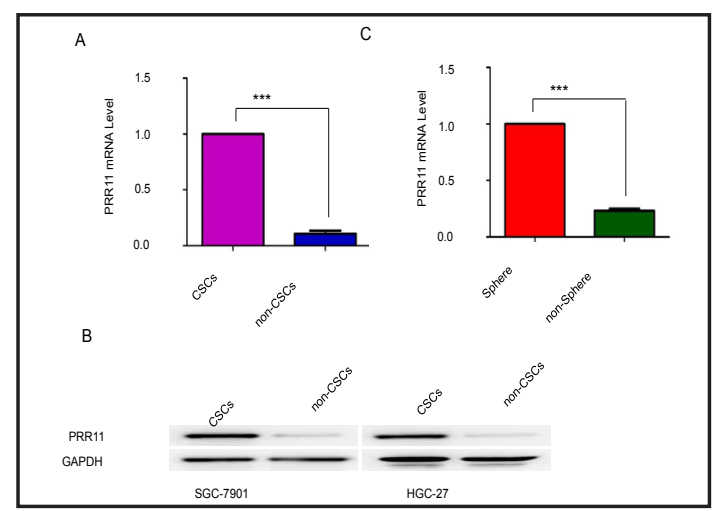


Fig. 2. PRR11 was required for the self-renewal and tumorigenicity maintenance of gastric CSCs (A) PRR11 protein levels were analyzed by Western blotting in SGC-7901 CSCs after infection with different shRNAs against PRR11. Sphere (B) and clone (C) formation assays of PRR11knockdown and scrambled-control in gastric CSCs from SGC-7901. Data are presented as mean \pm SEM of three independent experiments. ** $\mathrm{P}<0.01, \quad * * * \mathrm{P}<0.001 . \quad$ (D-F) Knock down of PRR11 expression

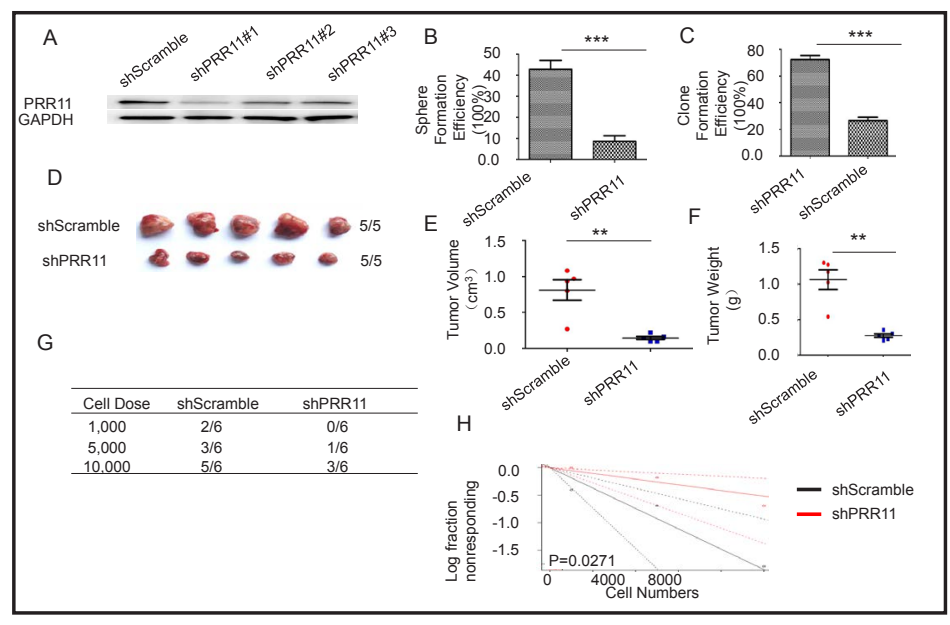
in gastric CSCs from SGC-7901 inhibited tumor growth in vivo. Data are presented as mean \pm SEM. $N=5$ for each group. ${ }^{* *} \mathrm{P}<0.01$. (G and H) Limiting dilution assay of gastric CSCs from SGC-7901 after infection with shScrambled or shPRR11 in NOD/SCID mice ( $\mathrm{N}=6$ for each group). CSC frequency was determined by the software ELDA (http://bioinf. wehi.edu.au/software/elda).

non-CSCs. In addition, we confirmed that the mRNA level of PRR11 was higher in sphere cells than in non-sphere cells $(\mathrm{P}<0.001$, Fig. 1C).

\section{PRR11 played roles in self-renewal and tumor-initiation of gastric CSCS}

We next investigated the potential role of PRR11 in self-renewal and tumorigenicity of gastric CSCs. We first examined whether knockdown of PRR11 expression in gastric CSCs could inhibit their self-renewal and tumorigenicity. Three shRNAs targeting PRR11 were designed and shPRR11\#1 exhibited the strongest reduction of PRR11 protein levels in gastric cancer cell line SGC-7901 (Fig. 2A), which was used in subsequent experiments. Knockdown of PRR11 expression in gastric CSCs significantly reduced the efficiency of sphere formation (42.1 \pm 3.8 vs. $9.7 \pm 1.2, \mathrm{P}<0.001$, Fig. $2 \mathrm{~B}$ ) and clone formation $(72.3 \pm 2.5$ vs. $29.8 \pm 1.3, \mathrm{P}<0.001$, Fig. 2C). We next established a xenograft tumor model using NOD/SCID mice to examine the role of PRR11 in tumor development. Compared with control gastric CSCs, PRR11-KD gastric CSCs required more cells and longer incubation time to generate tumor with equivalent size in vivo (Fig. 2D-2F). To quantify the tumor initiating capacity of PRR11-KD gastric CSCs, we transplanted cells at different cell numbers $\left(10^{3}, 5 \times 10^{3}\right.$ and $\left.10^{4}\right)$ per recipient. The incidence of tumors in mice receiving gastric CSCs was significantly higher than that in the PRR11KD gastric CSC cohorts (Fig. 2G). Limiting dilution analysis showed that the frequency was significantly higher in gastric CSCs compared with PRR11-KD counterparts ( $\mathrm{P}=0.0271$, Fig. $2 \mathrm{H}$ ). These findings suggest that a high level of PRR11 expression in gastric CSCs plays an essential role in self-renewal and tumorigenicity.

\section{Overexpression PRR11 rendered gastric non-CSCs to behave like CSCs}

We next infected gastric cancer cell lineSGC-7901 with lentiviral vector containing PRR11, and established PRR11 stably overexpressing cell line (Fig. 3A). PRR11 overexpression increased clone and sphere formation efficiencies significantly $(\mathrm{P}<0.05$, Fig. $3 \mathrm{~B}$ and $3 \mathrm{C})$. Tumor initiation assay showed that four out of five animals developed small tumors when gastric non-CSCs were implanted, whereas all animals developed large tumors when gastric non-CSCs expressing PRR11 were implanted (Fig. 3D). Tumor volume and tumor weight were significantly increased in animals implanted with gastric non-CSCs expressing PRR11 compared with liver non-CSCs $(\mathrm{P}<0.05$, Fig. $3 \mathrm{E}$ and $3 \mathrm{~F})$. These data further demonstrate that PRR11 plays a fundamental role in maintaining self-renewal of gastric CSCs. 
Fig. 3. PRR11 overexpression enhanced tumorigenic capacity of gastric non-CSCs. (A) NonCSC cell line SGC-7901 stably expressing PRR11 was established, and the expression of PRR11 was determined by western blotting. Efficiencies of sphere (B) and clone (C) formation were determined in non-CSCs expressing exogenous PRR11. Data are presented as mean \pm SEM of three independent experiments. ${ }^{*} \mathrm{P}<0.05$. (D-F) PRR11overexpressing or vector control treated non-CSCs were subcutaneously injected into NOD/SCID mice for observation of tumor growth. Results are shown as means \pm SEM. $\mathrm{N}=5$ for each group. ${ }^{*} \mathrm{P}<0.05$.

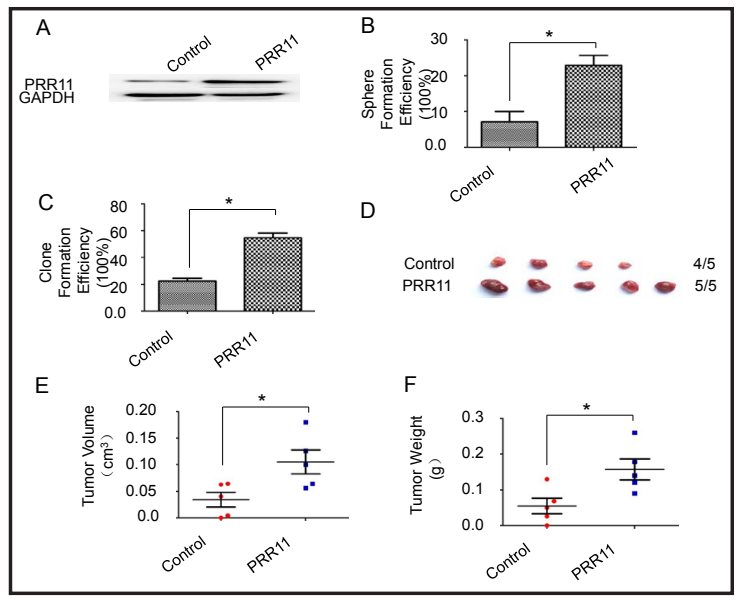

Fig. 4. PRR11 regulated stemness properties in gastric CSCs. (A and B) Expression of indicated stemness factors were measured by western blotting in CSCs or non-CSCs cell line after infected with shPRR11 (A) or PRR11 (B).

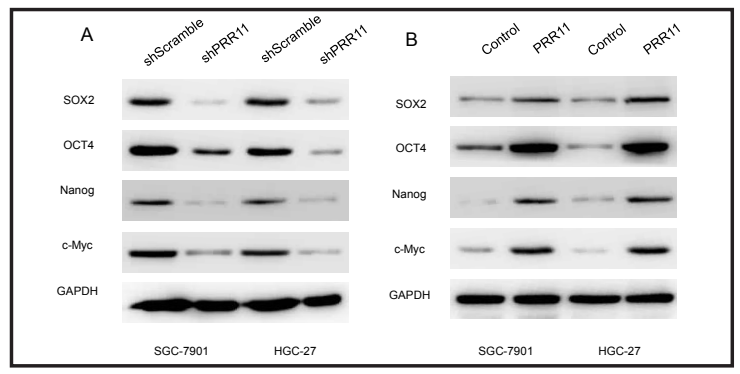

PRR11 regulated stemness properties in gastric CSCS

It has been reported that pluripotent transcription factors (SOX2, Oct4, Nanog, and c-Myc,) are involved in self-renewal of CSCs $[13,14]$. To explore the molecular mechanisms involved in PRR11 mediated self-renewal and tumorigenic potential of gastric CSCs, we first examined the expression of these genes in gastric CSCs upon PRR11 silencing. Our results showed that PRR11 knockdown in gastric CSCs decreased SOX2, Oct4, Nanog, and c-Myc expression levels (Fig. 4A). We also found that overexpressed PRR11 in gastric non-CSCs increased SOX2, Oct4, Nanog, and c-Myc expression levels (Fig. 4B). Together, these data suggest that PRR11 may regulate stemness transcriptional factors expression for priming self-renewal of gastric CSCs.

\section{MAPK signaling regulated the PRR11 protein stability of gastric CSCS}

It has been reported that MAPK signaling plays an important role in CSCs self-renewal and tumorigenicity [15-17]. However, how MAPK signaling sustains gastric CSCs self-renewal and tumorigenicity remains largely unknown. To investigate whether MAPK signaling affects PRR11 expression to enhance self-renewal of gastric CSCs, we treated gastric CSCs with the MAPK signaling inhibitor and measured PRR11 expression. Our results showed that PRR11 protein was significantly lower in gastric CSCs treated with MAPK signaling inhibitor Dorsomorphin (Fig. 5A), but not PRR11 mRNA levels (P>0.05, Fig. 5B).

We hypothesized that MAPK signaling may regulate the stability of the PRR11 protein. To do this, we measured PRR11 half-life in gastric CSCs treated with cyclohexamide (HCX), an inhibitor of protein translation. The half-life of PRR11 was short in gastric CSCs after treatment with MAPK signaling inhibitor Dorsomorphin (Fig. 5C). We treated gastric CSCs with the proteasome inhibitor MG132 with or without Dorsomorphin. Our results showed that treatment with MG132 could restore the reduced PRR11 protein level by Dorsomorphin (Fig. 5D). These results indicate that MAPK signaling may inhibit proteasome activity to maintain PRR11 protein stability. 


\section{Cellular Physiology Cell Physiol Biochem 2018;47:1721-1728 \begin{tabular}{l|l} 
DOI: 10.1159/000491005 & $\begin{array}{l}\text { O 2018 The Author(s). Published by S. Karger AG, Basel } \\
\text { www.karger.com/cpb }\end{array}$
\end{tabular} \\ Hu et al.: PRR11 and Gastric Cancer Stem Cells}

Fig. 5. MAPK signaling regulated the PRR11 protein stability of gastric CSCs. PRR11 protein levels (A) and mRNA levels (B) were measured in SGC-7901 CSCs by western blotting or QRT-PCR after treatment with different doses of Dorsomorphin. Data are presented as means \pm SEM from three independent experiments. (C) Endogenous PRR11 was examined by western blotting after treatment with $20 \mu \mathrm{g} /$ $\mathrm{mL}$ of cycloheximide (CHX) or cycloheximide plus Dorsomorphin. Cells were harvested at the indicated time points. (D) Gastric CSCs (SGC-7901 and HGC27) were treated with Dorsomorphin for 24 hours

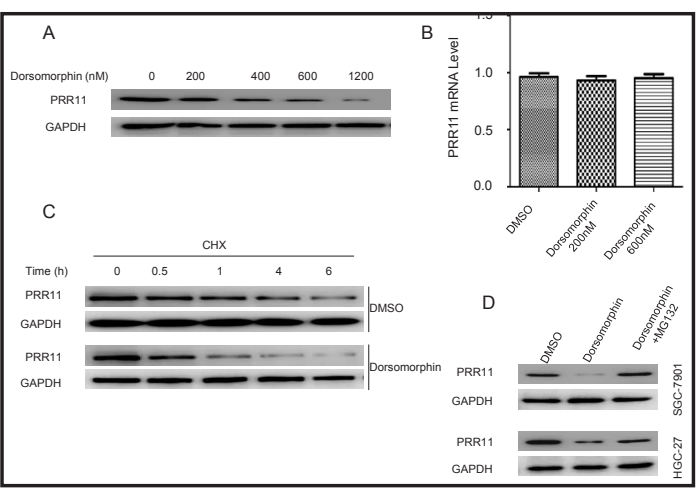
and then treated with $20 \mu \mathrm{M}$ MG-132 for 4 hours. Cell lysates were used for western blotting with the indicated antibodies.

Fig. 6. MAPK signaling maintained gastric CSCs selfrenewal dependent PRR11. Efficiencies of sphere (A) and clone (B) formation were determined in SGC-7901 CSCs after inhibiting MAPK signaling. Data are presented as mean \pm SEM of three independent experiments. ${ }^{*} \mathrm{P}<0.05,{ }^{*} \mathrm{P}<0.01,{ }^{*} * * \mathrm{P}<0.001$.

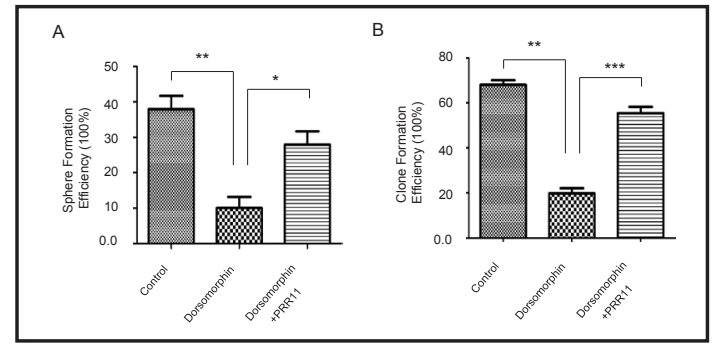

MAPK signaling maintained gastric CSCs self-renewal on a PRR11 dependent manner

Above results showed that PRR11 plays an important role in self-renewal and tumorigenicity of gastric CSCs, and MAPK regulates PRR11 protein stability. We expected that MAPK signaling though PRR11 may improve the self-renewal in gastric CSCs. To test this idea, we first overexpressed PRR11 in gastric CSCs in which MAPK signaling was inhibited by Dorsomorphin. Overexpression of exogenous PRR11 could restore the decreased clone and sphere formation efficiencies induced by inhibition of MAPK signaling in gastric CSCs (Fig. $6 \mathrm{~A}$ and $6 \mathrm{~B})$. These data demonstrate that MAPK signaling regulates gastric CSCs self-renewal on a PRR11 dependent manner.

\section{Discussion}

The CSCs possess critical roles in gastric cancer initiation, progression, and recurrence associated with resistance to radio- and chemotherapy [18]. In the present study, we showed that PRR11 was highly expressed in a subpopulation of gastric cancer cells with stem-like characteristics, and sat at the center of a signaling network, since changes in its expression pattern could drastically alter the biology properties of gastric CSCs and non-CSCs. The higher level of PRR11 expression in gastric CSCs appeared to be required to maintain the biological features of those cells, including self-renewal, stemness, and tumor initiation in vivo. Therefore, the pluripotent-like signature displayed by gastric CSCs could be at least partially attributed to the upregulation of PRR11. Moreover, we provided evidence that PRR11 possesses the capability to reprogram gastric non-CSCs with differentiated features to resemble CSCs-like status.

Because of CSCs are believed to up-regulation of multiple stemness genes to exert their regulatory effects on specific biological processes [19]. In this case, it was not surprising that we identified that knocked down or over-expression PRR11 affected stemness expression. However, the detailed mechanisms of PRR11 regulating pluripotency factors in gastric CSC need further exploration. 
A particularly interesting aspect of MAPK signaling in cancer progression is that the role of MAPK signaling may be linked to PRR11, which has emerged as a potential therapeutic target for cancers. In this study, our results suggested that MAPK signaling regulated the ability of PRR11 protein though the inhibition of ubiquitin-proteasome activity. This notion is clearly demonstrated by that the antagonist for MAPK signaling could block the self-renewal property of gastric CSCs. However, the stability of PRR11 itself is poorly understood, and further studies are required to determine whether MAPK signaling-related proteins bind directly to PRR11 itself or another protein involved in PRR11 degradation.

In addition, a rapidly growing body of evidence has shown PRR11 as potential targets for cancer therapy $[11,20]$. It has been reported that MAPK signaling was involved in a variety of cancers, such as lung cancer, pancreatic cancer, prostate cancer, melanoma and colon cancer $[21,22]$. MAPK signaling plays an important role in cancer formation, progression, therapy resistance and causing poor patient prognosis. Taken together, the striking inhibitory effect of PRR11 antagonists on tumor growth strongly suggests that further efforts toward development of anti-PRR11-based therapeutics are fully warranted.

\section{Acknowledgements}

This work was supported by The National Natural Science Fund of China (U1504816).

\section{Disclosure Statement}

The authors declare that they have no competing interests.

\section{References}

1 Huh YJ, Lee JH: The Advances of Laparoscopic Gastrectomy for Gastric Cancer. Gastroenterol Res Pract 2017;2017:9278469.

-2 Li J, Li Z, Zheng W, Li X, Wang Z, Cui Y, Jiang X: PANDAR: a pivotal cancer-related long non-coding RNA in human cancers. Mol Biosyst 2017;10.1039/c7mb00414a

-3 Tanaka M, Yoshimoto T, Nakamura T: A double-edged sword: the world according to Capicua in cancer. Cancer Sci 2017;10.1111/cas.13413

4 Zhang J, Huang T, Zhou Y, Cheng ASL, Yu J, To KF, Kang W: The oncogenic role of Epstein-Barr virus-encoded microRNAs in Epstein-Barr virus-associated gastric carcinoma. J Cell Mol Med 2017;10.1111/jcmm.13354

5 Krause M, Dubrovska A, Linge A, Baumann M: Cancer stem cells: Radioresistance, prediction of radiotherapy outcome and specific targets for combined treatments. Adv Drug Deliv Rev 2017;109:63-73.

6 Menendez JA, Corominas-Faja B, Cuyas E, Garcia MG, Fernandez-Arroyo S, Fernandez AF, Joven J, Fraga MF, Alarcon T: Oncometabolic Nuclear Reprogramming of Cancer Stemness. Stem Cell Reports 2016;6:273-283.

7 Zavros Y: Initiation and Maintenance of Gastric Cancer: A Focus on CD44 Variant Isoforms and Cancer Stem Cells. Cell Mol Gastroenterol Hepatol 2017;4:55-63.

8 Nguyen PH, Giraud J, Chambonnier L, Dubus P, Wittkop L, Belleannee G, Collet D, Soubeyran I, Evrard S, Rousseau B, Senant-Dugot N, Megraud F, Mazurier F, Varon C: Characterization of Biomarkers of Tumorigenic and Chemoresistant Cancer Stem Cells in Human Gastric Carcinoma. Clin Cancer Res 2017;23:1586-1597.

-9 Yang L, Xu JF, Kang Q, Li AQ Jin P, Wang X, He YQ Li N, Cheng T, Sheng JQ: Predictive Value of Stemness Factor Sox2 in Gastric Cancer Is Associated with Tumor Location and Stage. PLoS One 2017;12:e0169124.

10 Zhou F, Liu H, Zhang X, Shen Y, Zheng D, Zhang A, Lai Y, Li H: Proline-rich protein 11 regulates epithelial-tomesenchymal transition to promote breast cancer cell invasion. Int J Clin Exp Pathol 2014;7:8692-8699.

11 Wang Y, Zhang Y, Zhang C, Weng H, Li Y, Cai W, Xie M, Long Y, Ai Q, Liu Z, Du G, Wang S, Niu Y, Song F, Ozaki T, Bu Y: The gene pair PRR11 and SKA2 shares a NF-Y-regulated bidirectional promoter and contributes to lung cancer development. Biochim Biophys Acta 2015;1849:1133-1144. 


\section{Cellular Physiology Cell Physiol Biochem 2018;47:1721-1728 \begin{tabular}{l|l|l|}
\hline DOI: 10.1159/000491005 & $\begin{array}{l}\text { C) } 2018 \text { The Author(s). Published by S. Karger AG, Basel } \\
\text { www.karger.com/cpb }\end{array}$ \\
\hline
\end{tabular} \\ Hu et al.: PRR11 and Gastric Cancer Stem Cells}

12 Song Z, Liu W, Xiao Y, Zhang M, Luo Y, Yuan W, Xu Y, Yu G, Hu Y: PRR11 Is a Prognostic Marker and Potential Oncogene in Patients with Gastric Cancer. PLoS One 2015;10:e0128943.

13 Narayan S, Bryant G, Shah S, Berrozpe G, Ptashne M: OCT4 and SOX2 Work as Transcriptional Activators in Reprogramming Human Fibroblasts. Cell Rep 2017;20:1585-1596.

14 Thiagarajan PS, Zheng Q Bhagrath M, Mulkearns-Hubert EE, Myers MG, Lathia JD, Reizes O: STAT3 activation by leptin receptor is essential for TNBC stem cell maintenance. Endocr Relat Cancer 2017;24:415-426.

15 Mzoughi S, Zhang J, Hequet D, Teo SX, Fang H, Xing QR, Bezzi M, Seah MKY, Ong SLM, Shin EM, Wollmann H, Wong ESM, Al-Haddawi M, Stewart CL, Tergaonkar V, Loh YH, Dunn NR, Messerschmidt DM, Guccione E: PRDM15 safeguards naive pluripotency by transcriptionally regulating WNT and MAPK-ERK signaling. Nat Genet 2017;49:1354-1363.

16 Neiswender JV, Kortum RL, Bourque C, Kasheta M, Zon L, Morrison DK, Ceol CJ: KIT suppresses BRAFV600E-mutant melanoma by attenuating oncogenic RAS/MAPK signaling. Cancer Res 2017;10.1158/0008-5472.can-17-0473.

17 Yamagata K, Izawa Y, Onodera D, Tagami M: Chlorogenic acid regulates apoptosis and stem cell markerrelated gene expression in A549 human lung cancer cells. Mol Cell Biochem 2017;10.1007/s11010-0173171-1

18 Li L, Wei X, Wu B, Xiao Y, Yin M, Yang Q: siRNA-mediated knockdown of ID1 disrupts Nanog- and Oct-4mediated cancer stem cell-likeness and resistance to chemotherapy in gastric cancer cells. Oncol Lett 2017;13:3014-3024.

19 Courtois S, Duran RV, Giraud J, Sifre E, Izotte J, Megraud F, Lehours P, Varon C, Bessede E: Metformin targets gastric cancer stem cells. Eur J Cancer 2017;84:193-201.

20 Chen Y, Cha Z, Fang W, Qian B, Yu W, Li W, Yu G, Gao Y: The prognostic potential and oncogenic effects of PRR11 expression in hilar cholangiocarcinoma. Oncotarget 2015;6:20419-20433.

-21 Zhang C, Liu T, Wang G, Wang H, Che X, Gao X, Liu H: Rac3 Regulates Cell Invasion, Migration and EMT in Lung Adenocarcinoma through p38 MAPK Pathway. J Cancer 2017;8:2511-2522.

22 Ciuffreda L, Del Curatolo A, Falcone I, Conciatori F, Bazzichetto C, Cognetti F, Corbo V, Scarpa A, Milella M: Lack of growth inhibitory synergism with combined MAPK/PI3K inhibition in preclinical models of pancreatic cancer. Ann Oncol 2017;10.1093/annonc/mdx335. 